\title{
RECOVERY OF STOKES WAVES FROM VELOCITY MEASUREMENTS ON AN AXIS OF SYMMETRY
}

\author{
BOGDAN-VASILE MATIOC
}

\begin{abstract}
We provide a new method to recover the profile of Stokes waves, and more generally of waves with smooth vorticity, from measurements of the horizontal velocity component on a vertical axis of symmetry of the wave surface. Although we consider periodic waves only, the extension to solitary waves is straightforward.
\end{abstract}

\section{INTRODUCTION}

A Stokes wave is a two-dimensional periodic wave that travels at constant speed over a flat bed solely under the influence of gravity. The flow beneath a Stokes wave is irrotational and the surface profile is symmetric with respect to both crest and trough lines. Initiated by Stokes [30], the rigorous study of Stokes waves was developed steadily to uncover many fascinating aspects of these remarkable water flows, cf. e.g. [2, 6, 8, 11, 33].

The problem of reconstructing the free surface of regular Stokes waves from measured flow data, such as the pressure on the fluid bed, is of great importance in the field of fluid dynamics, cf. $[1,5,13,14,24,28,34]$. A very simple relation between the pressure on the bed and the elevation of the water surface is obtained when assuming that the pressure is hydrostatic, see the discussion in $[5,28]$. This method is used for tsunami detection, but, as it does not account for nonlinear wave effects, prediction errors are quite frequent [14]. On the other hand, the transfer function approach $[16,23]$ uses a linear formula relating the wave surface elevation to the pressure on the fluid bed, a large body of research being dedicated to determining a suitable transfer function. This latter method restricts primarily to linear irrotational waves. Therefore, it does not account for nonlinear effects and sometimes overestimates the height of large waves [34].

Within the exact theory of irrotational water waves, a nonlinear and nonlocal relation between the wave profile of solitary waves and the pressure on the bed was derived in $[13,28]$. An alternative exact local recovery formula was obtained in $[7,20]$. For a recovery formula in the setting of steady periodic waves we refer to $[4,5]$. In fact it is theoretically possible to recover the profile of solitary waves from the pressure on the bed even when allowing for rotational flows, provided that the vorticity distribution is real-analytic [19].

The goal of the present note is to present a new exact method to recover the profile of periodic traveling waves and the velocity field within the flow from measurements of the horizontal velocity on a vertical axis of symmetry of the wave. Our analysis is within the setting of rotational periodic waves with a smooth vorticity distribution, the extension to solitary waves is straightforward. In particular, our method can be used to reconstruct the free surface of Stokes waves. That the profile of rotational periodic gravity waves with a smooth vorticity is determined by the horizontal velocity

2010 Mathematics Subject Classification. 35J60; 76B07; 76B15.

Key words and phrases. water waves; recovery; horizontal velocity; 
on a vertical axis of symmetry - which is either a crest or a trough line - is quite intriguing and it underlines the strong connection between the flow and the free surface motion. This intricate connection is suggested also by the result of [26] where it is shown that the symmetry of the free wave surface can be characterized intrinsically in terms of the underlying flow.

The main tool used in our approach is a very recent observation pertaining to the regularity of rotational flows: any weak solution of the water wave problem possesses real-analytic streamlines, the radius of analyticity being independent of the position in the fluid, cf. [15]. Finally, we emphasize that the horizontal velocity on crest or trough lines has been measured in many laboratory experiments $[18,21,22,29,31]$ and therefore we expect that the practical accuracy of our recovery method can be verified numerically.

\section{Equivalent formulations of the Water WaVe PRoblem}

We consider steady periodic water waves propagating at the surface of a two-dimensional fluid body with wave speed $c>0$. The Cartesian coordinates $(x, y, z)$ are fixed such that the $x$-axis is the direction of wave propagation, the $y$-axis points vertically upwards, and the flow is independent of the $z$-coordinate. The fluid layer is bounded from below by the flat bed $y=-d$, whereby $d>0$. Neglecting viscosity effects and temperature variations, the motion of the fluid is governed by the Euler equations. As we consider steady waves only, the space-time dependence of the free surface, of the pressure, and of the velocity field has the form $(x-c t)$. Eliminating time by the change of coordinates $(x-c t, y, z) \mapsto(x, y, z)$, in the new coordinates system in which the origin moves in the direction of propagation of the wave with wave speed $c$, the wave is stationary and the flow is steady, the equations of motion being encompassed in the following system

$$
\left\{\begin{aligned}
(u-c) u_{x}+v u_{y} & =-P_{x} & & \text { in } \Omega_{\eta}, \\
(u-c) v_{x}+v v_{y} & =-P_{y}-g & & \text { in } \Omega_{\eta}, \\
u_{x}+v_{y} & =0 & & \text { in } \Omega_{\eta}, \\
P & =P_{0} & & \text { on } y=\eta(x), \\
v & =(u-c) \eta^{\prime} & & \text { on } y=\eta(x), \\
v & =0 & & \text { on } y=-d .
\end{aligned}\right.
$$

Hereby, $y=\eta(x)$ is the surface of the wave, $\Omega_{\eta}:=\{(x, y):-d<y<\eta(x)\}$ is the fluid domain, $P$ denotes the dynamic pressure, $u$ is the horizontal velocity, $v$ is the vertical velocity, $g$ is the gravity of Earth, the constant $P_{0}$ is the uniform air pressure, and the water's density is set to be $\rho=1$.

Classical solutions $(u, v, P, \eta)$ of $(2.1)$ belong to the following regularity class

$$
(u, v, P, \eta) \in\left(C_{p e r}^{1}\left(\bar{\Omega}_{\eta}\right)\right)^{3} \times C_{p e r}^{2}(\mathbb{R}),
$$

the subscript per being used to express the $\lambda$-periodicity of $(u, v, P, \eta)$ with respect to the variable $x$, and where the constant $\lambda>0$ is the wavelength. A last assumption we make is that the wave speed $c$ exceeds the horizontal velocity of each particle within the fluid

$$
\sup _{\Omega_{\eta}} u<c .
$$

The formulation (2.1) can be also recast in terms of the streamfunction $\psi$ which is defined by

$$
\psi(x, y):=-p_{0}+\int_{-d}^{y}(u(x, s)-c) d s \quad \text { for }(x, y) \in \bar{\Omega}_{\eta},
$$


the constant $p_{0}<0$, which represents the relative mass flux, being given by

$$
p_{0}:=\int_{-d}^{\eta(x)}(u(x, s)-c) d s .
$$

It follows from the third and the last two equations in (2.1) that $p_{0}$ is independent of $x$. Let us also observe that since $\nabla \psi=(-v, u-c)$, the vorticity of the flow is given by the scalar function $\omega:=u_{y}-v_{x}=\Delta \psi$.

Because of (2.3), the map $\mathcal{H}: \bar{\Omega}_{\eta} \rightarrow \bar{\Omega}$, with $\Omega:=\mathbb{R} \times\left(p_{0}, 0\right)$, defined by the relation

$$
\mathcal{H}(x, y):=(q, p)(x, y)=(x,-\psi(x, y)) \quad \text { for }(x, y) \in \bar{\Omega}_{\eta}
$$

is a diffeomorphism, that is $\mathcal{H} \in \operatorname{Diff}^{2}\left(\Omega_{\eta}, \Omega\right)$. Moreover, it follows from (2.1), cf. e.g. [25, Section $2]$, that $\omega \circ \mathcal{H}^{-1}=: \gamma$ depends only on the variable $p$. The function $\gamma \in C\left(\left[p_{0}, 0\right]\right)$ is the socalled vorticity function. Note that both $\omega$ and $\gamma$ are zero constant functions for Stokes flows. Using Bernoulli's law, we find that $(\eta, \psi) \in C_{p e r}^{2}(\mathbb{R}) \times C_{p e r}^{2}\left(\bar{\Omega}_{\eta}\right)$ solves the following free boundary problem

$$
\left\{\begin{aligned}
\Delta \psi & =\gamma(-\psi) & & \text { in } \Omega_{\eta}, \\
|\nabla \psi|^{2}+2 g(y+d) & =Q & & \text { on } y=\eta(x), \\
\psi & =0 & & \text { on } y=\eta(x), \\
\psi & =-p_{0} & & \text { on } y=-d,
\end{aligned}\right.
$$

with the positive constant $Q:=2\left(E-P_{0}\right)$. In terms of $\psi$ the condition $(2.3)$ appears as

$$
\sup _{\Omega_{\eta}} \psi_{y}<0 .
$$

For the existence theory, but also when studying the properties of solutions of $(2.7)$, cf. [12, 15], it is useful to express the hydrodynamical problem in terms of the height function $h: \bar{\Omega} \rightarrow \mathbb{R}$ that is defined by $h:=y \circ \mathcal{H}^{-1}+d$. The function $h \in C_{\text {per }}^{2}(\bar{\Omega})$ associates to each pair $(q, p) \in \bar{\Omega}$, the height of the water particle $(x, y):=\mathcal{H}^{-1}(q, p)$ above the bed. Particularly, we have that $\eta(x)=h(x, 0)-d$ for all $x \in \mathbb{R}$. It follows easily from the definition of $h$ and (2.7), cf. e.g. [12], that $h$ solves a quasilinear elliptic equation with nonlinear boundary conditions

$$
\left\{\begin{aligned}
\left(1+h_{q}^{2}\right) h_{p p}-2 h_{p} h_{q} h_{p q}+h_{p}^{2} h_{q q}-\gamma h_{p}^{3} & =0 & & \text { in } \Omega, \\
1+h_{q}^{2}+(2 g h-Q) h_{p}^{2} & =0 & & \text { on } p=0, \\
h & =0 & & \text { on } p=p_{0},
\end{aligned}\right.
$$

while the assumption (2.3) is now equivalent to

$$
\inf _{\Omega} h_{p}>0 .
$$

In the next Section, we use the equivalence of the three formulations within the set of classical solutions. The proof of this result follows easily from the arguments presented in [6, Chapter 3] and in $[25]$.

Proposition 2.1 (Equivalence of the three formulations). The following are equivalent:

(i) the equations (2.1) and (2.3) with $(u, v, P, \eta)$ satisfying (2.2);

(ii) the equations $(2.7)$ and $(2.8)$ with $(\eta, \psi) \in C_{p e r}^{2}(\mathbb{R}) \times C_{p e r}^{2}\left(\bar{\Omega}_{\eta}\right)$ and $\gamma \in C\left(\left[p_{0}, 0\right]\right)$;

(iii) the equations (2.9) and (2.10) with $h \in C_{\text {per }}^{2}(\bar{\Omega})$ and $\gamma \in C\left(\left[p_{0}, 0\right]\right)$. 


\section{THE MAIN RESUlT AND DISCUSSION}

In order to state our main result, we emphasize first that any (vertical) axis of symmetry of the wave must either be a trough or a crest line. This is a simple consequence of the real-analyticity of the wave profile, a property which holds a priori for solutions in the class (2.2), cf. [15, Corollary 1.2]. The main result of this paper is the following theorem.

Theorem 3.1. Consider a regular ${ }^{1}$ two-dimensional symmetric and periodic gravity wave with vorticity $\omega \in C^{\infty}\left(\bar{\Omega}_{\eta}\right)$ that travels over the flat bed $y=-d$ with wave speed $c>0$. Furthermore, assume that the horizontal component $u$ of the velocity field is known on the line of symmetry

$$
\left\{\left(x_{0}, y\right):-d \leq y \leq \eta\left(x_{0}\right)\right\}
$$

of the wave. Then, we can recover the wave profile, the velocity field and the pressure distribution within the flow.

Before proving Theorem 3.1 it is worthwhile to add some remarks.

Remark 3.2. (a) Theorem 3.1 is true in particular for Stokes waves for which the vorticity is zero. We emphasize that a constant nonzero vorticity, for which the assumption $\omega \in C^{\infty}\left(\bar{\Omega}_{\eta}\right)$ is also trivially satisfied, is relevant in many physical situations, cf. the discussion in [32].

(b) We remark that to the best of our knowledge, there is no rigorous proof for the existence of two-dimensional periodic steady gravity waves which are not symmetric. For criteria which ensure the symmetry of such waves with respect to both trough and crest lines we refer to $[9,10,26,27]$.

(c) In the proof of Theorem 3.1, we do not use of the assumption of periodicity. Particularly, our result shows that also the surface of solitary waves can be recovered when knowing the velocity field on a axis of symmetry.

(d) The values of $u$ on the vertical symmetry line $\left[x=x_{0}\right]$ and the wave speed are not both needed, but just the values of $u-c$ on this axis of symmetry.

(e) The proof of Theorem 3.1 uses to a large extent the fact that the streamlines of the flow are all real-analytic, the radius of analyticity being uniform in the fluid. An open question is whether the radius of analyticity can be chosen to be $\infty$.

Proof of Theorem 3.1. Let $\eta,(u, v)$, and $P$ be the unknown wave profile, velocity field, and pressure distribution, respectively. Because of the equivalence result in Proposition 2.1, we only need to determine the height function $h$. Observing that the problem (2.1) is invariant with respect to horizontal translations we may set $x_{0}=0$. Hence, $\eta$ is symmetric with respect to the vertical line $[x=0]$.

Our assumptions together with (2.5) enable us to determine the relative mass flux constant $p_{0}$, cf. (2.5). Knowing the strip $\Omega$ where the height function $h \in C_{p e r}^{2}(\bar{\Omega})$ is defined, we next show that $h$ is well-determined by the restriction of $(u-c)$ on the axis of symmetry $[x=0]$. To this end, we recall that $\Delta \psi=\omega$ in $\Omega_{\eta}$ and that $\psi$ is constant on $\partial \Omega_{\eta}$. From our assumption $\omega \in C^{\infty}\left(\bar{\Omega}_{\eta}\right)$ and the real-analyticity of the wave profile we deduce that $\psi \in C^{\infty}\left(\bar{\Omega}_{\eta}\right)$, cf. [17, Theorem 6.19]. Therewith, we get $\gamma=\omega \circ \mathcal{H}^{-1} \in C^{\infty}\left(\left[p_{0}, 0\right]\right)$ and also $h \in C_{p e r}^{\infty}(\bar{\Omega})$.

\footnotetext{
${ }^{1}$ By regular wave we mean a solution $(u, v, P, \eta)$ of the hydrodynamical problem (2.1) that satisfies (2.2) and (2.3). Notice that this class of solutions ensures that the three formulations are equivalent, cf. Proposition 2.1. The condition (2.3) is satisfied by regular Stokes waves automatically, only the Stokes wave of greatest height having the property that $u=c$ at the crest where the free water surface forms an angle of 120 degrees, cf. [33].
} 
Using the weak elliptic maximum principle in the context of (2.9) as in [26, Lemma 3.2] shows that the symmetry of the free water surface with respect to $[x=0]$, that is $\eta(x)=\eta(-x)$ for all $x \in \mathbb{R}$, also implies the symmetry of $h$, that is $h(q, p)=h(-q, p)$ for all $(q, p) \in \bar{\Omega}$. Even more holds: there exists a constant $L>0$ such that

$$
\left\|\partial_{q}^{m} h\right\|_{C^{2}(\bar{\Omega})} \leq L^{m-1}(m-2) ! \quad \text { for all } m \geq 2,
$$

cf. [3, Theorem 3.3] (see also [15] for a more general result). The estimate (3.1) ensures the realanalyticity of the streamlines as $[q \mapsto h(q, p)], p \in\left[p_{0}, 0\right]$, is real-analytic. Indeed, (3.1) implies for each $\left(q_{0}, p\right) \in \bar{\Omega}$ that

$$
\left|h(q, p)-\sum_{k=0}^{n} \frac{\partial_{q}^{k} h\left(q_{0}, p\right)}{k !}\left(q-q_{0}\right)^{k}\right| \leq \frac{\left\|\partial_{q}^{n+1} h\right\|_{0}}{(n+1) !}\left|q-q_{0}\right|^{n+1} \leq \frac{\left(L\left|q-q_{0}\right|\right)^{n+1}}{L} \rightarrow_{n \rightarrow \infty} 0
$$

if $L\left|q-q_{0}\right|<1$. In particular, the radius of convergence of the Taylor series is independent of the point $\left(q_{0}, p\right)$. This latter property enables us to extend the function $h$ to the whole strip $\bar{\Omega}$, provided that we know it on the subset $\left[-(2 L)^{-1} \leq q \leq(2 L)^{-1}\right] \subset \bar{\Omega}$, where the Taylor series of $[q \mapsto h(q, p)]$ converges for each $p \in\left[p_{0}, 0\right]$.

With this, our task reduces to determining $h$ in the rectangle $\left[-(2 L)^{-1} \leq q \leq(2 L)^{-1}\right]$. We note that the symmetry of $h$ implies in particular that $\partial_{q}^{2 n+1} h(0, p)=0$ for all $n \in \mathbb{N}$ and $p \in\left[p_{0}, 0\right]$. This property and (3.2) yield that

$$
h(q, p)=\sum_{n=0}^{\infty} a_{2 n}(p) q^{2 n} \quad \text { for }|q|<(2 L)^{-1} \text { and } p \in\left[p_{0}, 0\right],
$$

whereby $a_{2 n}:=((2 n) !)^{-1} \partial_{q}^{2 n} h(0, \cdot) \in C^{\infty}\left(\left[p_{0}, 0\right]\right)$ for all $n \in \mathbb{N}$. Similarly to (3.2), one can deduce from (3.1) that

$$
\begin{aligned}
& h_{p}(q, p)=\sum_{n=0}^{\infty} a_{2 n}^{\prime}(p) q^{2 n}, \quad h_{q}(q, p)=\sum_{n=1}^{\infty} 2 n a_{2 n}(p) q^{2 n-1}, \quad h_{p p}(q, p)=\sum_{n=0}^{\infty} a_{2 n}^{\prime \prime}(p) q^{2 n}, \\
& h_{q p}(q, p)=\sum_{n=1}^{\infty} 2 n a_{2 n}^{\prime}(p) q^{2 n-1}, \quad h_{q q}(q, p)=\sum_{n=1}^{\infty} 2 n(2 n-1) a_{2 n}(p) q^{2 n-2}
\end{aligned}
$$

for $\left|q-q_{0}\right| \leq(2 L)^{-1}$ and all $p \in\left[p_{0}, 0\right]$.

Since the values of $(u-c)$ on the symmetry line $[x=0]$ are known, we can determine the coefficient function $a_{0}$ as follows. From the definition of $h$ we obtain that $h(0,-\psi(0, y))=y+d$ for all $y \in[-d, \eta(0)]$, whereby, in virtue of $(2.4)$ and (2.5),

$$
\psi(0, y)=-p_{0}+\int_{-d}^{y}(u(0, s)-c) d s, \quad y \in[-d, \eta(0)] .
$$

Because of $(2.8)$, the map $-\psi(0, \cdot):[-d, \eta(0)] \rightarrow\left[p_{0}, 0\right]$ is bijective and its inverse is thus also known. With the notation from Section 2 it is the function $y \circ \mathcal{H}^{-1}(0, \cdot):\left[p_{0}, 0\right] \rightarrow[-d, \eta(0)]$. Hence, $a_{0}$ is determined by the formula $a_{0}=y \circ \mathcal{H}^{-1}(0, \cdot)+d:\left[p_{0}, 0\right] \rightarrow[0, d+\eta(0)]$.

To finish the proof, we show that $a_{0}$ determines all the other coefficient functions $a_{2 n}, n \in \mathbb{N}$, $n \geq 1$. Indeed, plugging the Taylor expansions (3.2) and (3.3) into the first equation of (2.9) yields, after identifying for a fixed $p \in\left[p_{0}, 0\right]$ the coefficients of each power of $q$, that

$$
A_{0}+C_{0}+D_{0}=0 \quad \text { and } \quad A_{2 n}+B_{2 n}+C_{2 n}+D_{2 n}=0 \quad \text { for } n \geq 1 .
$$


Hereby, we used the following relations

$$
\left(1+h_{q}^{2}\right) h_{p p}=\sum_{n=0}^{\infty} A_{2 n} q^{2 n}, \quad-2 h_{q} h_{p} h_{p q}=\sum_{n=1}^{\infty} B_{2 n}, \quad q^{2 n} h_{p}^{2} h_{q q}=\sum_{n=0}^{\infty} C_{2 n} q^{2 n}, \quad-\gamma h_{p}^{3}=\sum_{n=0}^{\infty} D_{2 n} q^{2 n}
$$

with

$$
\begin{aligned}
& A_{2 n}=a_{2 n}^{\prime \prime}+\sum_{k=1}^{n} a_{2(n-k)}^{\prime \prime}\left(\sum_{l=1}^{k} 4 l(k-l+1) a_{2 l} a_{2(k-l+1)}\right), \\
& B_{2 n}=-2 \sum_{k=0}^{n-1} a_{2(n-k-1)}^{\prime}\left(\sum_{l=1}^{k+1} 4 l(k-l+2) a_{2 l} a_{2(k-l+2)}^{\prime}\right), \\
& C_{2 n}=\sum_{k=0}^{n}(2 k+1)(2 k+2) a_{2 k+2}\left(\sum_{l=0}^{n-k} a_{2 l}^{\prime} a_{2(n-k-l)}^{\prime}\right), \\
& D_{2 n}=-\gamma \sum_{k=0}^{n} a_{2 k}^{\prime}\left(\sum_{l=0}^{n-k} a_{2 l}^{\prime} a_{2(n-k-l)}^{\prime}\right) .
\end{aligned}
$$

The first equation of (3.5) is equivalent to

$$
a_{2}=\frac{\gamma a_{0}^{\prime}}{2}-\frac{a_{0}^{\prime \prime}}{2\left(a_{0}^{\prime}\right)^{2}}
$$

whereby, in view of $u<c$ in $\bar{\Omega}_{\eta}$, we have $a_{0}^{\prime}>0$. Moreover, from the second equation of (3.5) we find that

$$
\begin{aligned}
a_{2 n+2}= & \frac{1}{(2 n+1)(2 n+2)\left(a_{0}^{\prime}\right)^{2}}\left[-a_{2 n}^{\prime \prime}-\sum_{k=1}^{n} a_{2(n-k)}^{\prime \prime}\left(\sum_{l=1}^{k} 4 l(k-l+1) a_{2 l} a_{2(k-l+1)}\right)\right. \\
& +2 \sum_{k=0}^{n-1} a_{2(n-k-1)}^{\prime}\left(\sum_{l=1}^{k+1} 4 l(k-l+2) a_{2 l} a_{2(k-l+2)}^{\prime}\right) \\
& \left.-\sum_{k=0}^{n-1}(2 k+1)(2 k+2) a_{2 k+2}\left(\sum_{l=0}^{n-k} a_{2 l}^{\prime} a_{2(n-k-l)}^{\prime}\right)+\gamma \sum_{k=0}^{n} a_{2 k}^{\prime}\left(\sum_{l=0}^{n-k} a_{2 l}^{\prime} a_{2(n-k-l)}^{\prime}\right)\right]
\end{aligned}
$$

for all integers $n \geq 1$. The relations (3.6) and (3.7) show that $a_{2 n+2}$ is uniquely determined by $a_{0}, \ldots, a_{0}^{(2 n)}, \gamma, \ldots, \gamma^{(2 n)}$. This completes the proof.

Acknowledgements The author thanks the anonymous referees for the comments and suggestions which have improved the quality of the article.

\section{REFERENCES}

[1] A. Baquerizo and M. A. Losada. Transfer function between wave height and wave pressure for progressive waves. Coastal Engineering, 24:351-353, 1995.

[2] B. Buffoni and J. Toland. Analytic Theory of Global Bifurcation. Princeton Series in Applied Mathematics. Princeton University Press, Princeton, NJ, 2003. An introduction. 
[3] H. Chen, W.-X. Li, and L.-J. Wang. Regularity of traveling free surface water waves with vorticity. J. Nonlinear Sci., 23:1111-1142, 2013.

[4] D. Clamond. New exact relations for easy recovery of steady wave profiles from bottom pressure measurements. J. Fluid Mech., 726:547-558, 2013.

[5] D. Clamond and A. Constantin. Recovery of steady periodic wave profiles from pressure measurements at the bed. J. Fluid Mech., 714:463-475, 2013.

[6] A. Constantin. Nonlinear Water Waves with Applications to Wave-Current Interactions and Tsunamis, volume 81 of CBMS-NSF Conference Series in Applied Mathematics. SIAM, Philadelphia, 2011.

[7] A. Constantin. On the recovery of solitary wave profiles from pressure measurements. J. Fluid Mech., 699:376$384,2012$.

[8] A. Constantin. Mean velocities in a Stokes wave. Arch. Ration. Mech. Anal., 207(3):907-917, 2013.

[9] A. Constantin, M. Ehrnström, and E. Wahlén. Symmetry of steady periodic gravity water waves with vorticity. Duke Math. J., 140(3):591-603, 2007.

[10] A. Constantin and J. Escher. Symmetry of steady periodic surface water waves with vorticity. J. Fluid Mech., 498(1):171-181, 2004.

[11] A. Constantin and J. Escher. Analyticity of periodic traveling free surface water waves with vorticity. Ann. of Math., 173:559-568, 2011.

[12] A. Constantin and W. Strauss. Exact steady periodic water waves with vorticity. Comm. Pure Appl. Math., 57(4):481-527, 2004.

[13] B. Deconinck, D. Henderson, K. L. Oliveras, and V. Vasan. Recovering the water-wave surface from pressure measurements. In Proc. 10th Intl Conf. on Waves, Vancouver Juli 25-29, 2011, pages 699-702. PIMS, 2011.

[14] M. Donelan and W. Hui. Mechanics of Ocean Surface Waves. In G. Geernaert and W. Plant, editors, Surface Waves and Fluxes, volume 7 of Environmental Fluid Mechanics, pages 209-246. Springer Netherlands.

[15] J. Escher and B.-V. Matioc. On the analyticity of periodic gravity water waves with integrable vorticity function. Differential Integral Equations, 27(3-4):217-232, 2014.

[16] J. Escher and T. Schlurmann. On the recovery of the free surface from the pressure within periodic traveling water waves. J. Nonlinear Math. Phys., 15(suppl. 2):50-57, 2008.

[17] D. Gilbarg and N. S. Trudinger. Elliptic Partial Differential Equations of Second Order. Springer Verlag, 2001.

[18] J. Grue, D. Clamond, M. Huseby, and A. Jensen. Kinematics of extreme waves in deep water. Applied Ocean Research, 25:355-366, 2003.

[19] D. Henry. On the pressure transfer function for solitary water waves with vorticity. Math. Ann., 357(1):23-30, 2013.

[20] H.-C. Hsu. Recovering surface profiles of solitary waves on a uniform stream from pressure measurements. Discrete Contin. Dyn. Syst. Ser. A, 34(8):3035-3043, 2014.

[21] H.-C. Hsu, Y.-Y. Chen, C.-Y. Lin, and C.-Y. Cheng. Experimental study of the velocity field in solitary water waves. Journal of Nonlinear Mathematical Physics, 19(supp01):1240003, 2012.

[22] Y. Iwagaki and T. Sakai. Horizontal water particle velocity of finite amplitude waves. Proc. Twelfth Conf. Coastal Eng., pages 309-325, 1970.

[23] P. K. Kundu and I. M. Cohen. Fluid Mechanics. Academic Press, 2010.

[24] Y.-Y. Kuo and Y.-F. Chiu. Transfer function between wave height and wave pressure for progressive waves. Coastal Engineering, 23:81-93, 1994.

[25] B.-V. Matioc. Analyticity of the streamlines for periodic traveling water waves with bounded vorticity. Int. Math. Res. Not., 17:3858-3871, 2011.

[26] B.-V. Matioc. A characterization of the symmetric steady water waves in terms of the underlying flow. Discrete Contin. Dyn. Syst. Ser. A, 34(8):3125-3133, 2014.

[27] H. Okamoto and M. Shōji. The Mathematical Theory of Permanent Progressive Water-Waves. Adv. Ser. Nonlinear Dynam. 20. World Scientific Pub Co Inc, 2001.

[28] K. L. Oliveras, V. Vasan, B. Deconinck, and D. Henderson. Recovering the water-wave profile from pressure measurements. SIAM J. Appl. Math., 72(3):897-918, 2012.

[29] D. J. Skyner, W. J. Easson, and C. A. Greated. The internal kinematics of steep waves on sheared currents. Technical report, 1992. Dept. of Energy report, OTH 92366.

[30] G. G. Stokes. Mathematical and Physical Papers. Cambridge University Press, Cambridge, 1880. 
[31] C. Swan, I. P. Cummings, and R. L. James. An experimental study of two-dimensional surface water waves propagating on depth-varying currents. Part 1. Regular waves. J. Fluid Mech., 428:273-304, 2001.

[32] A. F. Teles da Silva and D. H. Peregrine. Steep, steady surface waves on water of finite depth with constant vorticity. J. Fluid Mech., 195:281-302, 1988.

[33] J. F. Toland. Stokes waves. Topol. Methods Nonlinear Anal., 7(1):1-48, 1996.

[34] C.-H. Tsai, M.-C. Huang, F.-J. Young, Y.-C. Yin, and H.-W. Li. On the recovery of surface wave by pressure transfer function. Ocean Engineering, 32:1247-1259, 2005.

Institut für Angewandte Mathematik, Leibniz Universität Hannover, Welfengarten 1, 30167 HanNOVER, Deutschland.

E-mail address: matioc@ifam.uni-hannover.de 